\title{
Flow cytometry for the evaluation of anti- plasmodial activity of drugs on Plasmodium falciparum gametocytes
}

\author{
Séverine Chevalley ${ }^{1,2}$, Agnès Coste ${ }^{1,2}$, Alexandrine Lopez ${ }^{1,2}$, Bernard Pipy ${ }^{3}$, Alexis Valentin ${ }^{1,2^{*}}$
}

\begin{abstract}
Background: The activity of promising anti-malarial drugs against Plasmodium gametocytes is hard to evaluate even in vitro. This is because visual examination of stained smears, which is commonly used, is not totally convenient. In the current study, flow cytometry has been used to study the effect of established anti-malarial drugs against sexual stages obtained from W2 strain of Plasmodium falciparum. Gametocytes were treated for $48 \mathrm{~h}$ with different drug concentrations and the gametocytaemia was then determined by flow cytometry and compared with visual estimation by microscopy.

Results and conclusions: Initially gametocytaemia was evaluated either using light microscopy or flow cytometry. A direct correlation $\left(r^{2}=0.9986\right)$ was obtained. Two distinct peaks were observed on cytometry histograms and were attributed to gametocyte populations. The activities of established anti-malarial compounds were then measured by flow cytometry and the results were equivalent to those obtained using light microscopy. Primaquine and artemisinin had $\mathrm{IC}_{50}$ of $17.6 \mu \mathrm{M}$ and $1.0 \mu \mathrm{M}$, respectively.

Gametocyte sex was apparently distinguishable by flow cytometry as evaluated after induction of exflagellation by xanthurenic acid. These data form the basis of further studies for developing new methods in drug discovery to decrease malaria transmission.
\end{abstract}

\section{Background}

Many different approaches have been used to measure the susceptibility of asexual stages of Plasmodium falciparum to anti-malarial drugs in vitro. The most used methods are light microscopy, which enables visual quantification of parasitized red blood cells, and radioactive methods that evaluate the viability of parasites by tracking the incorporation of ${ }^{3} \mathrm{H}$-hypoxanthine into their nucleic acids. However, only the first method allows the quantification of gametocytes, since these cells do not multiply. Given that light microscopy is labour-intensive, subjective, and suffers from interoperator variations, alternative methods for the counting of gametocytes are needed to increase the efficiency of drug-screening on this stage of the parasite's life-cycle.

\footnotetext{
* Correspondence: valentin@cict.fr

'Université de Toulouse-3, LPSNPR (Laboratoire Pharmacochimie des

Substances Naturelles et Pharmacophores Redox), 118 route de Narbonne, F31062 Toulouse cedex 9, France
}

In earlier studies, flow cytometry has been proposed to assess the viability of intra-erythrocytic stages of Plasmodium using DNA fluorescent stains [1,2]. Many fluorescent stains have been used in flow cytometry studies: propidium iodide [3], acridine orange [4] and YOYO-1 [5] require fixation and permeabilization of the parasites before use. Other dyes need complete lysis of erythocytes, such as Hoechst 33258 [6,7] and Picogreen ${ }^{\circ}$ [8], while hydroethine (HE) does not require lysis or fixatives. Plasmodium takes up and metabolizes HE into ethidium, a nucleic acid-binding fluorochrome [9]. Some authors have also proposed to combine thiazole orange with HE [10] or with Hoechst 33342 [11] to stain nucleic acids in order to differentiate intra-erythrocytic stages of $P$. falciparum.

In the present study, the discrimination, by flow cytometry, of $P$. falciparum asexual and sexual forms stained with HE was performed in order to validate a method for the screening of gametocytocidal drugs. First, the relative distribution of fluorescence in parasite 
subpopulations according to their maturity and sex was analyzed and then the gametocytocidal activity of drugs was determined. Finally, particular gametocyte preparations were treated with xanthurenic acid to follow the exflagellation process.

Flow cytometry was demonstrated to be usable to evaluate the different stages of sexual and asexual parasite populations and to assess the in vitro gametocytocidal activities of potentially anti-plasmodial drugs. Moreover, this method enables to discriminate the male and female gametocyte subpopulations.

\section{Methods}

Materials

Red blood cells and human serum were obtained from EFS, Toulouse (France); PBS, RPMI and additives were from Lonza (Belgium). All other reagents were from Sigma-Aldrich, l'Isle d'Abeau (France).

\section{Plasmodium falciparum in vitro culture}

Parasites were cultured according to Trager and Jensen [12], and synchronized according to Lambros [13]. Briefly, parasites were routinely maintained in $\mathrm{O}+$, human erythrocytes (parasitaemia: 0.5-4\%, haematocrit: 4\%) in RPMI 1640 with $25 \mathrm{mM}$ HEPES, $2 \mathrm{mM}$ L-glutamine and $7 \%$ human $\mathrm{AB}$ serum in a $\mathrm{CO}_{2}$ incubator.

Gametocyte cultures were initiated with W2-Indochina strain as described elsewhere [14], with modifications [15]. Cultures were treated with $50 \mathrm{mM} \mathrm{N}$-acetylD-glucosamine for 4-5 days to remove most of the asexual stages. Old (stage IV-V, 11-13-days-old) gametocyte cultures were mostly used.

Visual counting of schizonts and gametocytes was carried out on Giemsa-stained smears before and after concentration. Images were digitally recorded with a digital camera (DS Camera Control Unit DS-U2, Nikon) mounted on an optical microscope (Nikon Eclipse 80i, Nikon).

\section{Purification}

Magnetic purification was carried out by using the haemozoin paramagnetic complex property. Prior to purification, $\mathrm{MACS}^{\ominus}$ (25LD columns, Miltenyi BioTec, Germany) columns were filled with warmed $\left(37^{\circ} \mathrm{C}\right)$ RPMI. The experimentation was performed under sterile conditions $[16,17]$. The tested blood from cultures was then loaded on the columns (typically, $4 \mathrm{~mL}$ at $25-50 \%$ haematocrit) and warm $\left(37^{\circ} \mathrm{C}\right)$ culture medium was then added until the eluent was apparently free of red blood cells. At this point, the columns were removed from the magnetic support after addition of $10 \mathrm{~mL}$ more culture medium and the eluent was recovered. It was then centrifuged (800 g, $10 \mathrm{~min}$ ) and supernatant was discarded. The pellet was used to prepare blood smears that were
Giemsa-stained and it was also analysed by flow cytometry.

\section{In vitro tests with gametocytes}

Primaquine and artemisinin (the latter being kindly provided by Pierre Fabre Laboratories) were dissolved in DMSO, while chloroquine was dissolved in RPMI. Twelve day-old gametocyte cultures were transferred to 24-well or 96-well plates, and increasing dilutions of each drug were added. All experiments were performed in triplicate. After $48 \mathrm{~h}$ of incubation at $37^{\circ} \mathrm{C}$, thin blood smears were prepared and stained with Giemsa. The number of gametocytes per 10,000 erythrocytes was visually estimated by optical microscopy. In parallel, parasitaemia was evaluated by flow cytometry.

\section{Flow cytometry}

Cultures were washed with PBS. Pellets were resuspended in hydroethidine solution $(50 \mu \mathrm{g} / \mathrm{mL})$ for 20 minutes at $37^{\circ} \mathrm{C}$ in the dark. After washing in PBS, $10^{5}$ cells were analysed with a FACScalibur cytometer (Becton Dickinson ${ }^{\circ}$ ) using the CellQuestPro ${ }^{\circ}$ program for data analysis.

\section{Confocal microscopy}

In order to obtain an homogenous film, glass cover-slips were coated overnight at room temperature with polyL-lysine (1:100 in PBS). Parasite cultures were washed twice in PBS and the pellets were resuspended in $50 \mu \mathrm{g} /$ $\mathrm{mL}$ hydroethidine (HE) for 20 minutes at $37^{\circ} \mathrm{C}$ in the dark. Samples were then washed twice in PBS and resuspended in $1 \mathrm{~mL}$ of PBS. Cells were distributed on the poly-L-lysine-coated cover-slips and incubated for 20 minutes at $37^{\circ} \mathrm{C}$ in the dark. After cell adhesion, the cover-slips were washed with PBS and analysed with a fluorescence microscope (ConfoCor, Zeiss LM510, Carl Zeiss).

\section{In vitro exflagellation assay}

The exflagellation of gametocytes was quantified according to Kawamoto et al [18]. Gametocytes were highly purified on $\mathrm{MACS}^{\circ}$ columns and immediately resuspended at $5 \%$ final haematocrit in $100 \mu \mathrm{M}$ xanthurenic acid (XA) for $20 \mathrm{~min}$ at room temperature [19,20]. Prior to flow cytometry analysis, a visual counting of gametocytes with or without exflagellation was carried out on Giemsa-stained smears.

\section{Results and Discussion}

In vitro cultures were incubated with HE, which is converted to ethidium by metabolically active cells [9]. The interaction between ethidium and parasite nucleic acids resulted in a red fluorescence emission allowing discrimination between uninfected and infected erythrocytes 
independently of the parasitic stage (asexual stages: Figure 1; gametocytes: Figure 2). Histograms (left panels, Figures $1 \mathrm{~A}, 1 \mathrm{C}$ and $2 \mathrm{~A}$ ) and dot plots (right panels, Figures $1 \mathrm{~B}, 1 \mathrm{D}$ and $2 \mathrm{~B}$ ) show the distribution of HE fluorescence (x-axis, log scale). Plasmodium falciparum W2 strain cultures were synchronized by $5 \% \mathrm{D}$-sorbitol lysis to obtain ringenriched cultures [13], and by the magnetic enrichment method [16] in order to purify schizonts (Figure 1E) or gametocytes (Figure 2). The labelled M1 peak corresponded to non-fluorescent events (mostly uninfected erythrocytes, damaged erythrocytes and those with dead parasites inside). The fluorescence intensity of the rings was addressed to M2 peak (Figure 1A). We observed one peak M3, corresponding to late trophozoites and mature schizonts (Figure 1C). Schizonts exhibited an increase in fluorescence intensity when compared to the other stages.
These results were in line with those of Pace and Staalsoe $[21,22]$. Flow cytometry analysis of enriched gametocytes showed two distinct peaks of fluorescence (M2 and M3) (Figure 2A). Similar results were reported in a study on Green Fluorescence Protein (GFP) fluorescence of transgenic A-SET/GFP murine Plasmodium berghei gametocytes [21].

Enrichment of the W2 strain at a very high yield was obtained with magnetic columns [16] with an enrichment greater than 150-fold for the schizont enriched population. For example, in one experimentation, parasitaemia (mostly late trophozoites and mature schizonts) was $0.53 \%$ before purification with $\mathrm{MACS}^{\odot}$ and $83.04 \%$ after (M3 population, Figure $1 \mathrm{C}$ ). In another experimentation, gametocytaemia was $1.43 \%$ before and $13.7 \%$ after purification with $\mathrm{MACS}^{\oplus}$ (population, Figure 2A).

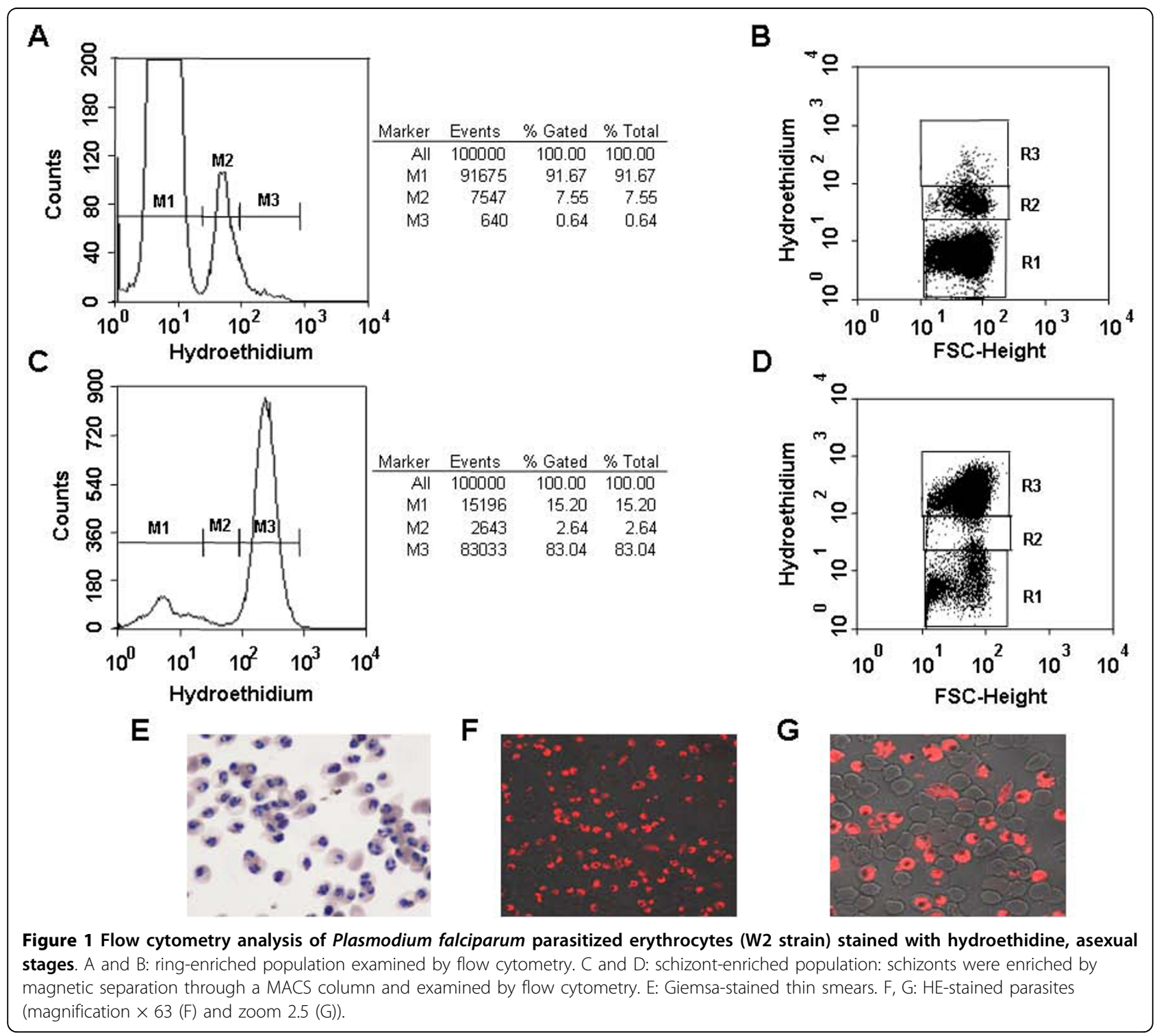


These results were similar to those obtained by numeration on Giemsa stained thin smears (Figures 1E, $2 \mathrm{C}$ and 2D). Similar results were observed throughout six independent experiments (Table 1). A true direct correlation $\left(\mathrm{r}^{2}=0.9805\right)$ was obtained between gametocytaemia determined by the two methods. More, the M2 population was, most of the times, lower than M3 (Table 1).

The repartition of gametocytes in each population (M2 and M3) could be due to the sex of the gametocytes, which modifies their amount of DNA, and/or to their stage of maturation. To determine whether the M2 and M3 peaks corresponded to the difference between male and female gametocytes, we compared gametocyte cultures before and after male gametogenesis. Exflagellation has been achieved by decreasing drastically the culture temperature (from $37^{\circ} \mathrm{C}$ to $23^{\circ} \mathrm{C}$ ) and submitting the gametocytes to xanthurenic acid (XA), a gametocyte-activating factor (GAF) $[23,24]$. The sensitivity of $P$. falciparum microgametocytes to XA was determined in vitro [19] and the exflagellation response was expressed as a percentage of a $100 \mu \mathrm{M} \mathrm{XA}$ control. The effector concentration for half-maximal response $\left(\mathrm{EC}_{50}\right)$ in cultured $P$. falciparum NF54 strain was $2 \mu \mathrm{M}$. In the current study, after enrichment by MACS ${ }^{\circ}$, a fraction of the purified gametocytes (strain W2) was treated with $100 \mu \mathrm{M} \mathrm{XA}$, a concentration that triggers the highest stimulatory effect on in vitro exflagellation [19]. This XA-stimulated culture was compared with the non-stimulated gametocytes by flow

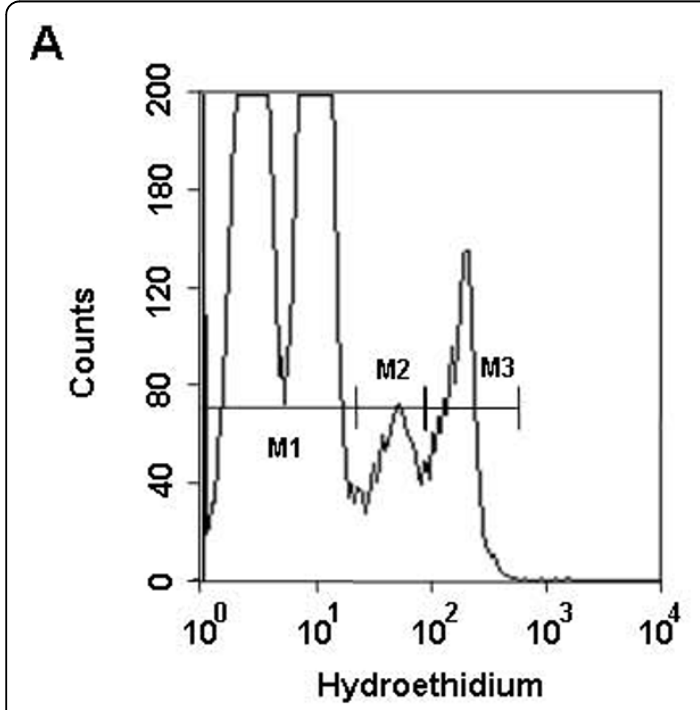

\begin{tabular}{rrrr} 
Marker & Events & $\%$ Gated & $\%$ Total \\
\hline All & 100000 & 100.00 & 100.00 \\
M2 & 6646 & 6.65 & 6.65 \\
M1 & 85134 & 85.13 & 85.13 \\
M3 & 8540 & 8.54 & 8.54
\end{tabular}

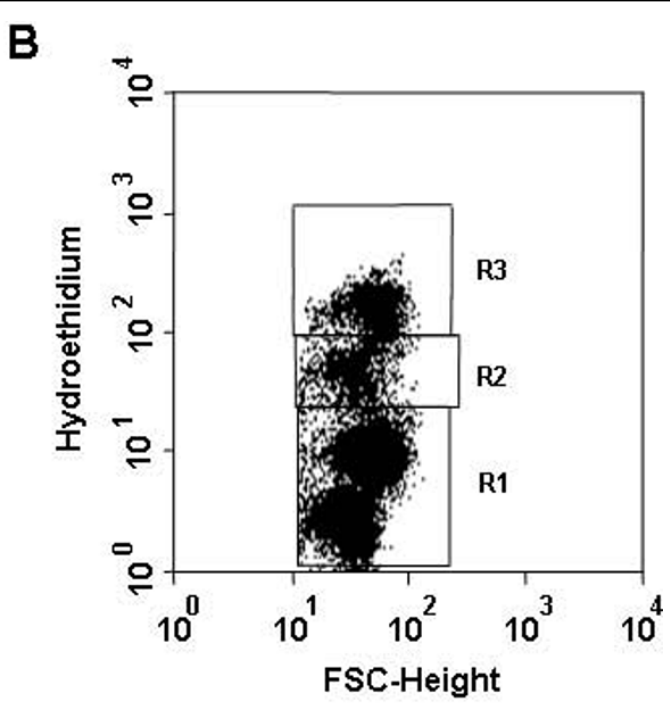

C

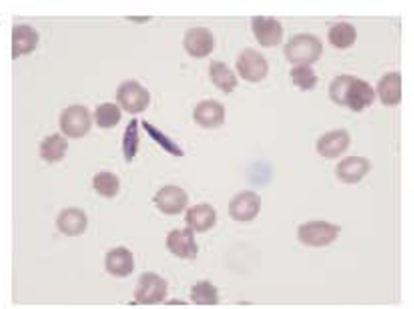

E

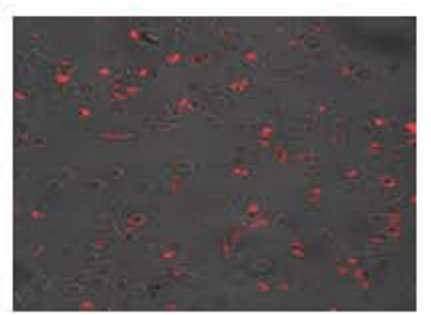

D

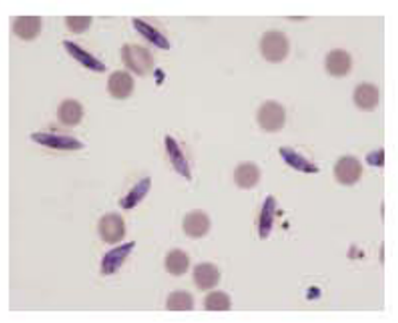

F

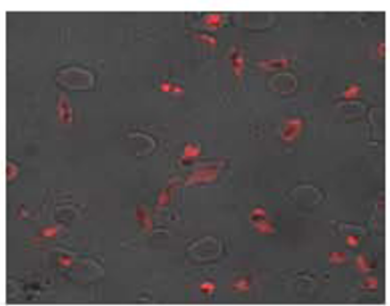

Figure 2 Flow cytometry analysis of Plasmodium falciparum parasitized erythrocytes (W2 strain) stained with hydroethidine, gametocytes. Gametocytes were purified by magnetic separation through a MACS column and examined by flow cytometry (A and B). Giemsastained thin smears (D) and HE-stained enriched culture (magnification $\times 63$ (E) and zoom 2.5 (F)). C: Giemsa-stained thin smears before gametocyte enrichment. 
Table 1 Comparison between FCA (Flow cytometry analysis) an OM (Optical microscopy) for the determination of gametocytaemia.

\begin{tabular}{cccccc}
\hline $\begin{array}{c}\text { Experiment } \\
\text { number }\end{array}$ & $\begin{array}{c}\text { M1 } \\
\text { (\%) }\end{array}$ & $\begin{array}{c}\text { Female } \\
\text { Gam } \\
\text { (\%): M2 }\end{array}$ & $\begin{array}{c}\text { Male Gam } \\
\text { + Sch (\%): } \\
\text { M3 }\end{array}$ & $\begin{array}{c}\text { Gam } \\
\text { (\%) }\end{array}$ & $\begin{array}{c}\text { Sch } \\
(\%)\end{array}$ \\
\hline 1 & 36.9 & 14.7 & 47.9 & 74 & 2.1 \\
\hline 2 & 66.9 & 2.5 & 28.7 & 38 & 19 \\
\hline 3 & 85.1 & 6.6 & 8.5 & 18.9 & 0.5 \\
\hline 4 & 80.9 & 12.6 & 6.4 & 13.4 & 0 \\
\hline 5 & 83.3 & 3.5 & 13.3 & 17 & 0 \\
\hline 6 & 90.9 & 5.8 & 2.5 & 6.9 & 1 \\
\hline 7 & 93.6 & 1.6 & 4.6 & 3 & 1 \\
\hline mean & 76.8 & 6.8 & 16.0 & 24.4 & 3.3 \\
\hline
\end{tabular}

Parasitaemias of in vitro $P$. falciparum gametocyte cultures (W2 strain) were evaluated by flow cytometry and optical microscopy (FCA: flow cytometry assay; OM: optical microscopy). These values correspond to the percentages of every population after gametocyte (Gam) enrichment by MACS.

M1: percentage corresponding to the first peak of the histogram in FCA, which corresponds to non-fluorescent events (uninfected erythrocytes). M2: parasitaemia corresponding to the second peak of the histogram in FCA (erythrocytes infected by gametocytes).

M3: parasitaemia corresponding to the third peak of the histogram in FCA (erythrocytes infected by gametocytes and/or residual asexual stages (schizonts: Sch)).

cytometry. The data are presented in Figure 3. Firstly, a slight increase of gametocytaemia was observed in the M2 population. Secondly, the exflagellation by the addition of XA decreased the gametocytaemia only in the M3 peak. The large error observed (Figure 3) can be attributed to the variability in the parasitaemia levels observed in the three independent experiments represented. Altogether these data showed that the decrease in the M3 peak after XA corresponded to a decrease in male gametocytes and hence showed that in the M2 population mostly female ones were present, unlike M3 population that contained mature male gametocytes and residual schizonts (Table 1). On one hand, the observation that the M2 population was less fluorescent (one female gametocyte leads to a single gamete) than the M3 populations, including numerous male gametocytes which leads to the formation of eight gametes was in agreement with the study by Pace et al [21]. These authors demonstrated that GFP fluorescence of late female gametocytes, identified by the sex-specific antibody Pfg377, was less intense than the fluorescence generated by male gametocytes [21]. On the other hand, the percentage of female gametocytes was higher than the percentage of mature male ones (Figure 3). These results were in accordance with sex ratio studies in P. falciparum that have showed that female gametocytes were generally more numerous than males. Generally, one male was observed for four females, though large fluctuations in the gametocyte sex ratio have been observed [25].

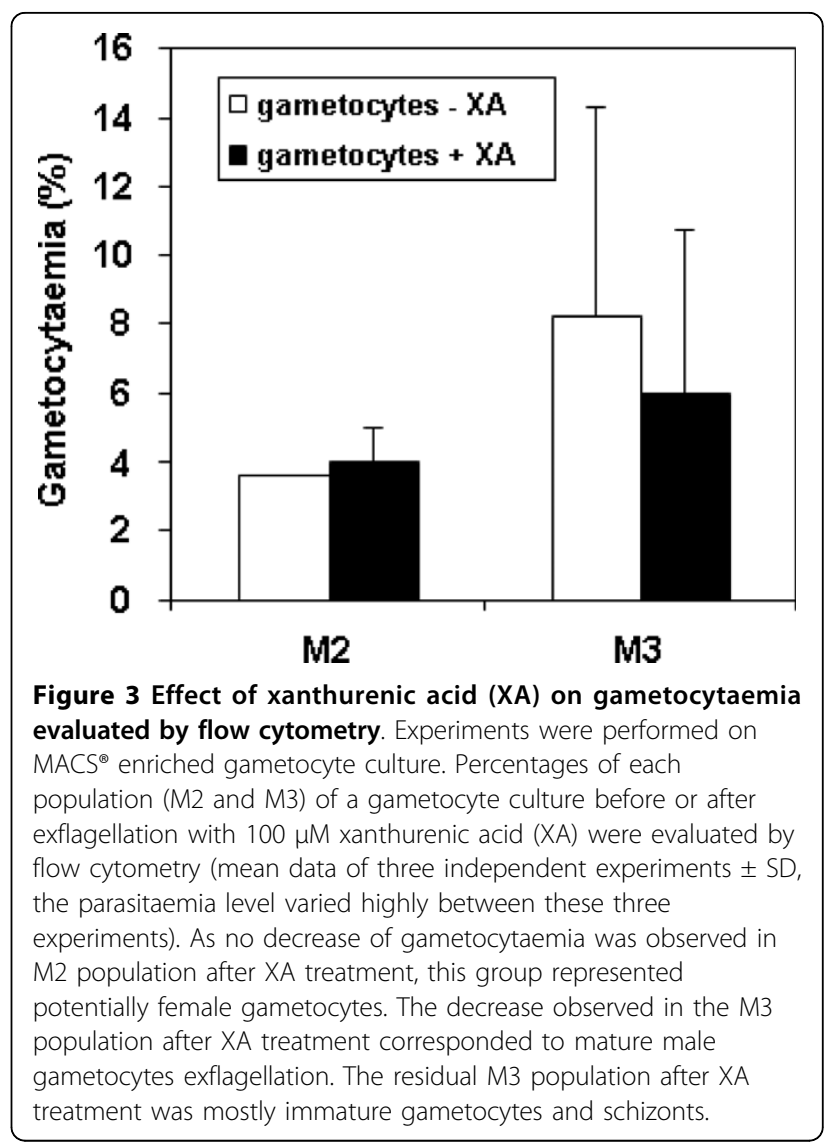

To validate the use of flow cytometry in order to evaluate the in vitro gametocytocidal activity of drugs, the efficiency of primaquine, artemisinin and chloroquine against gametocytes were compared both by the classic optical microscopy (OM) method and by FCA. After 12 days of maturation and treatment with $\mathrm{N}$ acetyl-D-glucosamine ( 5 days before testing) to remove most of asexual stages, gametocytes obtained from chloroquine-resistant W2 strain were exposed to these established anti-malarial compounds at increasing dilutions for $48 \mathrm{~h}$. The results are summarized in Table 2 . As expected, artemisinin and primaquine proved to be transmission-blocking drugs (artemisinin $\mathrm{IC}_{50}$ against gametocytes was $1,000 \mathrm{nM}$ by FCA and $690 \mathrm{nM}$ by $\mathrm{OM})$. As expected, chloroquine was inactive $\left(\mathrm{IC}_{50}>\right.$ $20,000 \mathrm{nM}$ by FCA and by OM). Primaquine, which had an $\mathrm{IC}_{50}$ against gametocytes of $17,600 \mathrm{nM}$ by FCA and $11,200 \mathrm{nM}$ by OM could be compared with gametocytaemia evaluated by OM by Sall et al (namely $9600 \mu \mathrm{M}$ [26]). Interestingly, the results obtained with the two methods (OM and FCA) were similar for the three anti-malarial compounds. Therefore, drug effects on gametocytaemia, which are usually analysed by conventional microscopy, could clearly be assessed by the flow cytometric method. 
Table 2 Conventional drugs activity against $P$. falciparum gametocytes.

\begin{tabular}{ccc}
\hline & FCA & OM \\
\hline Primaquine & 17.60 & 11.20 \\
\hline Artemisinin & 1.00 & 0.69 \\
\hline Chloroquine & $>20$ & $>20$ \\
\hline
\end{tabular}

In vitro anti-malarial activity of primaquine, artemisinin and chloroquine against $P$. falciparum gametocytes (W2 strain) evaluated by flow cytometry and optical microscopy (FCA: flow cytometry assay; OM: optical microscopy) (means of the data of three experiments). Values are given in $\mu \mathrm{M}$.

\section{Conclusions}

FCA, in combination with magnetic enrichment, has here been shown to be useful to estimate the inhibitory concentrations of known drugs against $P$. falciparum gametocytes and hence should be useful to evaluate promising anti-gametocyte drugs. Moreover, HE-labelled viable parasites, which were the only labelled cells, while Giemsa staining did not allow the differentiation between living and dead parasites. Although these results were obtained on $P$. falciparum, they are close to those of other authors [21,27] who have quantified GFP expression during intraerythrocytic development of transgenic A-SET/GFP P. berghei. They showed that the fluorescence intensity of gametocytes was comparable to that of late trophozoites, as showed in the present report. Using flow cytometry, other researchers have identified $P$. falciparum gametocytes well before they were morphologically distinguishable from asexual stage parasites, thanks to the use of the chimeric Pfs 16-GFP [28]. This FACS based assay used one of the earliest known gametocyte proteins, the Pfs 16 as a reporter. However, the present method can be used for nongenetically modified parasites.

\section{Acknowledgements}

The authors thank Nancy Voissiere for technical assistance and Antoine Berry and Françoise Benoit-Vical for fruitful discussions.

\section{Author details}

${ }^{1}$ Université de Toulouse-3, LPSNPR (Laboratoire Pharmacochimie des Substances Naturelles et Pharmacophores Redox), 118 route de Narbonne, F31062 Toulouse cedex 9, France. ${ }^{2}$ IRD, LPSNPR, F-31062 Toulouse, France. ${ }^{3}$ EA2405 UMR3 MD-UM-UPS, Université Paul Sabatier Toulouse III, France.

\section{Authors' contributions}

SC conceived and carried out the studies, drafted the manuscript. AC conceived the study, interpreted cytometry studies, redaction and correction of the manuscript. AL master degree student in the laboratory, participated to in vitro culture and cytometry studies. BP interpreted cytometry studies, drafted and corrected the manuscript. AV conceived the study and participated in its design and coordination, redaction and correction of the manuscript.

All authors read and approved the final manuscript.

\section{Competing interests}

The authors declare that they have no competing interests.
Received: 16 September 2009

Accepted: 11 February 2010 Published: 11 February 2010

\section{References}

1. Azas N, Rathelot P, Djekou S, Delmas F, Gellis A, Di Giorgio C, Vanelle P, Timon-David P: Antiparasitic activity of highly conjugated pyrimidine-2,4dione derivatives. Farmaco 2003, 58:1263-1270.

2. Wyatt CR, Goff W, Davis WC: A flow cytometric method for assessing viability of intraerythrocytic hemoparasites. J Immunol Meth 1991, 140:23-30.

3. Contreras CE, Rivas MA, Dominguez J, Charris J, Palacios M, Bianco NE, Blanca I: Stage-specific activity of potential antimalarial compounds measured in vitro by flow cytometry in comparison to optical microscopy and hypoxanthine uptake. Memorias do Instituto Oswaldo Cruz 2004, 99:179-184.

4. Bhakdi SC, Sratongno P, Chimma P, Rungruang T, Chuncharunee A, Neumann HP, Malasit P, Pattanapanyasat K: Re-evaluating acridine orange for rapid flow cytometric enumeration of parasitemia in malaria-infected rodents. Cytometry 2007, 71:662-667.

5. Li Q, Gerena L, Xie L, Zhang J, Kyle D, Milhous W: Development and validation of flow cytometric measurement for parasitemia in cultures of P. falciparum vitally stained with YOYO-1. Cytometry A 2007, 71:297-307.

6. Bianco AE, Battye FL, Brown GV: Plasmodium falciparum: rapid quantification of parasitemia in fixed malaria cultures by flow cytometry. Expl Parasitol 1986, 62:275-282.

7. van Vianen $\mathrm{PH}$, van Engen $\mathrm{A}$, Thaithong $\mathrm{S}$, Keur van der M, Tanke $\mathrm{HJ}$, Kaay van der HJ, Mons B, Janse CJ: Flow cytometric screening of blood samples for malaria parasites. Cytometry 1993, 14:276-280.

8. Corbett Y, Herrera L, Gonzalez J, Cubilla L, Capson TL, Coley PD, Kursar TA, Romero LI, Ortega-Barria E: A novel DNA-based microfluorimetric method to evaluate antimalarial drug activity. Am J Trop Med Hyg 2004, 70:119-124.

9. Heyde van der HC, Elloso MM, Waa vande J, Schell K, Weidanz WP: Use of hydroethidine and flow cytometry to assess the effects of leukocytes on the malarial parasite Plasmodium falciparum. Clin Diagn Lab Immunol 1995, 2:417-425.

10. Jouin H, Daher W, Khalife J, Ricard I, Puijalon OM, Capron M, Dive D: Double staining of Plasmodium falciparum nucleic acids with hydroethidine and thiazole orange for cell cycle stage analysis by flow cytometry. Cytometry 2004, 57:34-38.

11. Grimberg BT, Erickson JJ, Sramkoski RM, Jacobberger JW, Zimmerman PA: Monitoring Plasmodium falciparum growth and development by UV flow cytometry using an optimized Hoechst-thiazole orange staining strategy. Cytometry 2008, 73:546-554.

12. Trager W, Jensen JB: Human malaria parasites in continuous culture. Science 1976, 193:673-675.

13. Lambros C, Vanderberg JP: Synchronization of Plasmodium falciparum erythrocytic stages in culture. J Parasitol 1979, 65:418-420.

14. Ifediba T, Vanderberg JP: Complete in vitro maturation of Plasmodium falciparum gametocytes. Nature 1981, 294:364-366.

15. Benoit-Vical F, Lelievre J, Berry A, Deymier C, Dechy-Cabaret O, Cazelles J, Loup C, Robert A, Magnaval JF, Meunier B: Trioxaquines are new antimalarial agents active on all erythrocytic forms, including gametocytes. Antimicrob Agents Chemother 2007, 51:1463-1472.

16. Ribaut C, Berry A, Chevalley S, Reybier K, Morlais I, Parzy D, Nepveu F, Benoit-Vical F, Valentin A: Concentration and purification by magnetic separation of the erythrocytic stages of all human Plasmodium species. Malar J 2008, 7:45.

17. Trang DT, Huy NT, Kariu T, Tajima K, Kamei K: One-step concentration of malarial parasite-infected red blood cells and removal of contaminating white blood cells. Malar J 2004, 3:7.

18. Kawamoto F, Alejo-Blanco R, Fleck SL, Kawamoto Y, Sinden RE: Possible roles of $\mathrm{Ca} 2+$ and CGMP as mediators of the exflagellation of Plasmodium berghei and Plasmodium falciparum. Mol Biochem Parasitol 1990, 42:101-108.

19. Arai M, Billker O, Morris HR, Panico M, Delcroix M, Dixon D, Ley SV, Sinden RE: Both mosquito-derived xanthurenic acid and a host bloodderived factor regulate gametogenesis of Plasmodium in the midgut of the mosquito. Mol Biochem Parasitol 2001, 116:17-24. 
20. Bhattacharyya MK, Kumar N: Effect of xanthurenic acid on infectivity of Plasmodium falciparum to Anopheles stephensi. Int J Parasitol 2001, 31:1129-1133

21. Pace T, Olivieri A, Sanchez M, Albanesi V, Picci L, Siden Kiamos I, Janse CJ, Waters AP, Pizzi E, Ponzi M: Set regulation in asexual and sexual Plasmodium parasites reveals a novel mechanism of stage-specific expression. Mol Microbiol 2006, 60:870-882.

22. Staalsoe T, Giha HA, Dodoo D, Theander TG, Hviid L: Detection of antibodies to variant antigens on Plasmodium falciparum-infected erythrocytes by flow cytometry. Cytometry 1999, 35:329-336.

23. Billker $\mathrm{O}$, Lindo V, Panico M, Etienne AE, Paxton T, Dell A, Rogers $\mathrm{M}$, Sinden RE, Morris HR: Identification of xanthurenic acid as the putative inducer of malaria development in the mosquito. Nature 1998, 392:289-292.

24. Garcia GE, Wirtz RA, Barr JR, Woolfitt A, Rosenberg R: Xanthurenic acid induces gametogenesis in Plasmodium, the malaria parasite. J Biol Chem 1998, 273:12003-12005.

25. Talman AM, Domarle O, McKenzie FE, Ariey F, Robert V:

Gametocytogenesis: the puberty of Plasmodium falciparum. Malar J 2004, 3:24.

26. Sall C, Yapi AD, Desbois N, Chevalley S, Chezal JM, Tan K, Teulade JC, Valentin A, Blache Y: Design, synthesis, and biological activities of conformationally restricted analogs of primaquine with a 1,10phenanthroline framework. Bioorganic \& Medicinal Chemistry Letters 2008, 18:4666-4669.

27. Khan SM, Franke-Fayard B, Mair GR, Lasonder E, Janse CJ, Mann M, Waters AP: Proteome analysis of separated male and female gametocytes reveals novel sex-specific Plasmodium biology. Cell 2005, 121:675-687.

28. Dixon MW, Peatey CL, Gardiner DL, Trenholme KR: A green fluorescent protein-based assay for determining gametocyte production in Plasmodium falciparum. Mol Biochem Parasitol 2009, 163:123-126.

doi:10.1186/1475-2875-9-49

Cite this article as: Chevalley et al:. Flow cytometry for the evaluation of anti-plasmodial activity of drugs on Plasmodium falciparum gametocytes. Malaria Journal 2010 9:49.

\section{Submit your next manuscript to BioMed Central and take full advantage of:}

- Convenient online submission

- Thorough peer review

- No space constraints or color figure charges

- Immediate publication on acceptance

- Inclusion in PubMed, CAS, Scopus and Google Scholar

- Research which is freely available for redistribution

Submit your manuscript at www.biomedcentral.com/submit 